\title{
Risk score for the prediction of severe obesity in pediatric craniopharyngiomas: relative to tumor origin
}

\author{
Danling $\mathrm{Li}^{1}$, Jun Pan ${ }^{2}$, Junxiang Peng ${ }^{2}$, Shichao Zhang ${ }^{2}$, Guanglong Huang ${ }^{2}$, Xi'an Zhang ${ }^{2}$, Yun Bao ${ }^{2}$ and Songtao Qi ${ }^{2}$
}

BACKGROUND: We aimed to develop a risk score to improve the prediction of severe obesity in pediatric craniopharyngiomas (PCS).

METHODS: Overall, 612 consecutive PCs were prospectively enrolled from six hospitals. Data from 404 participants were analyzed. Participants from three of the six hospitals $(n=290)$ were used to develop a risk score. External validation of the developed risk score was conducted using the participants from the other three hospitals $(n=114)$. Sequential logistic regression was used to develop and validate the risk score. The c statistic and a calibration plot were used to assess the discrimination and calibration of the proposed risk score.

RESULTS: The overall frequency of severe obesity was $16.1 \%$ (65/404). The risk score employed a scale of 0-16 and demonstrated good discriminative power, with an optimismcorrected c statistic of 0.820 . Similar results were obtained from external validation, with a c statistic of 0.821 . The risk score showed good calibration, with no apparent over- or under-prediction observed in the calibration plots.

CONCLUSIONS: This novel risk score is a simple tool that can help clinicians assess the risk of severe obesity in PCs, thereby helping to plan and initiate the most appropriate disease management for these patients in time.

$\mathbf{P}$ ediatric craniopharyngiomas (PCs) are challenging and complex benign tumors of the sellar and suprasellar region. Because of their benign nature, total surgical removal with appropriate additional endocrine replacement therapy is the treatment of choice to prevent recurrence and improve patients' life quality $(1,2)$. However, their close proximity to vital neurovascular structures poses a significant surgical challenge, even in experienced hands. Hypothalamic obesity is a significant complication of craniopharyngiomas and is associated with increased mortality $(3,4)$.

Although several studies have sought to identify risk factors for the early detection of obesity before surgery (5-8), success has been variable, suggesting that a single risk factor is not demonstrated to be sufficient for evaluation in the clinical setting. Therefore, the application of a predictive model using multiple markers is likely to be of greater use. The primary goal of our study was to incorporate multiple clinical risk factors in the prediction of severe obesity in the early stages of PC. More specifically, we aimed to develop and validate a risk score that can identify patients with either a very high or a very low probability of developing severe obesity, using only baseline characteristics before a detectable change in serum creatinine, in PC patients.

\section{METHODS}

A total of 612 consecutive PC patients, aged 4-16 years, were prospectively enrolled between September 2006 and August 2012 from six regional central hospitals located in five cities in China. All participants gave informed consent for this specific study. Among these participants, 208 were excluded based on predetermined exclusion criteria (Figure 1). The remaining 404 participants comprised our study population, of whom 290 participants from three of the six hospitals served as the development cohort to develop the risk score. The remaining 114 participants from the other three hospitals served as an independent validation cohort.

All of the pathological materials and medical records of these patients were reviewed. The diagnosis of PC was confirmed with histology. The average age of the patients (260 male and 144 female patients) was $8.25 \pm 6.20$ years at the time of surgery (range 4-16 years). All of the clinical, radiological, and surgical data were recorded in detail. Tumor size was estimated as the maximum measurable dimension on magnetic resonance imaging and classified as small $(\leq 2 \mathrm{~cm})$, moderate $(2-4 \mathrm{~cm})$, large $(4-6 \mathrm{~cm})$, or huge $(>6 \mathrm{~cm})$. All 404 patients in this series underwent surgical resection with the aim of total tumor removal along the edge of the tumor membrane; the surgeries were performed by Professor Songtao Qi.

\section{Growth Pattern of PCs and Clinical Classification}

As indicated in our previous study, the pituitary stalk penetrates two membranous structures (namely, the diaphragma sellae and the basal arachnoid membrane) to reach the pituitary gland. Accordingly, PCs originating along the developmental path of the stalk may manifest different topographic relationships with these membranes. On the basis of comprehensive assessment of pre-surgical MR imaging, intra-operative findings, and the most likely originating site (9), tumors were classified into three subtypes, as illustrated in Figure 2 (type Q: infradiaphragmatic; type S: supradiaphragmatic, interarachnoid, extraventricular; and type T: supradiaphragmatic, infundibulotuberal). This topographical classification scheme highlights the site of origin of the tumor and the relationship between the tumor and supra-sellar membranous structures. Such a classification could serve as one of the criteria for surgical planning

${ }^{1}$ Department of Biostatistics, School of Public Health, Southern Medical University, Guangzhou, Guangdong, China; ${ }^{2}$ Department of Neurosurgery, Nanfang Hospital, Southern Medical University, Guangzhou, Guangdong, China. Correspondence: Songtao Qi (sjwkqisongtao@126.com)

The first five authors contributed equally to this work.

Received 9 April 2017; accepted 7 October 2017; advance online publication 20 December 2017. doi:10.1038/pr.2017.289 


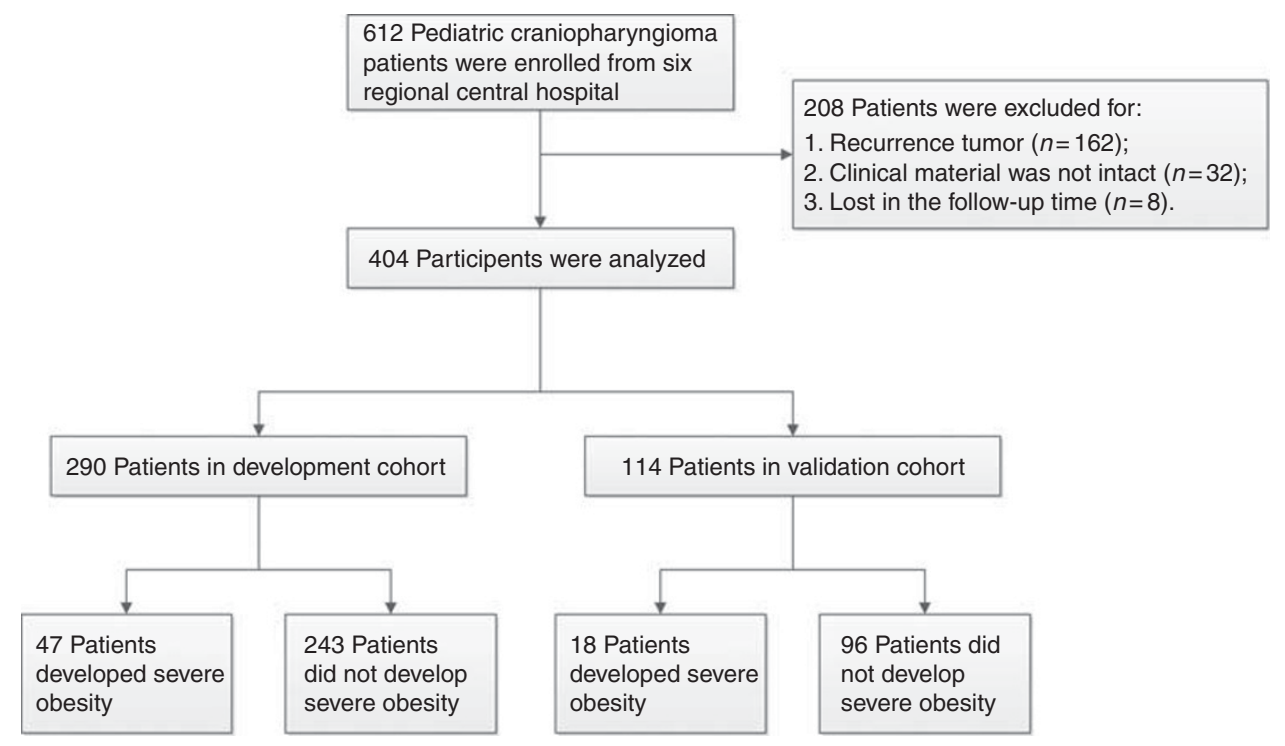

Figure 1. Flowchart of study participant enrollment.

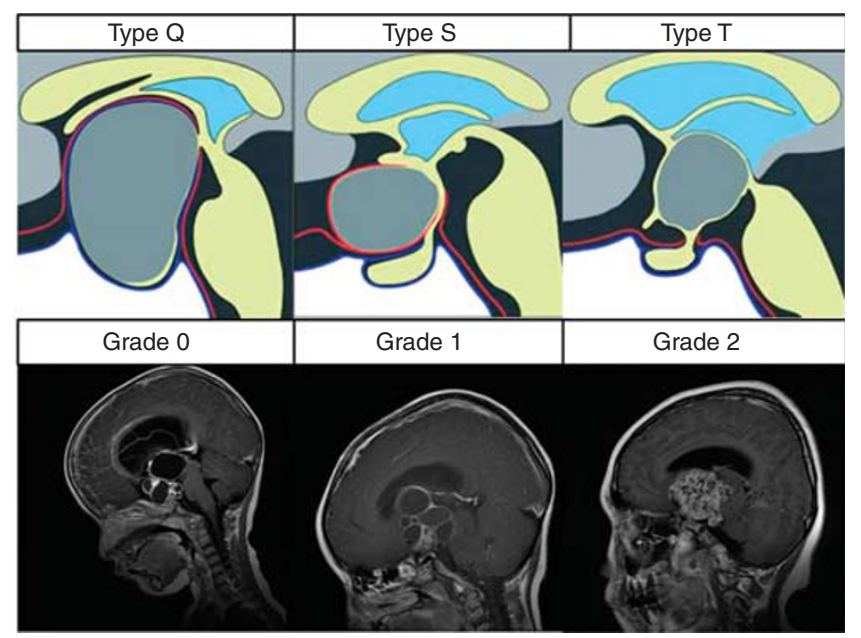

Figure 2. Topographical classification scheme illustrating growth patterns based on tumor origin (first row of graph) and PHS of PC patients: Grade 0: no hypothalamic damage; Grade 1: minimal hypothalamic damage; Grade 2: severe hypothalamic damage (second row of graph). PC, pediatric craniopharyngioma; PHS, preoperative hypothalamic status.

and broaden our understanding of these formidable tumors. On the basis of the type of tumor, we identified three distinct patterns of hypothalamic involvement before surgical resection of PCs. Preoperative hypothalamic status (PHS) at the time of diagnosis was evaluated using the assessment method according to Puget et al. (4) (Figure 2).

\section{Endocrinological Assessment}

Morning fasting venous blood samples were obtained from all subjects to detect plasma thyrotropin, free thyroxine (FT4 and FT3), cortisol, total testosterone, follicle stimulating hormone, luteinizing hormone, prolactin, growth hormone, and insulin-like growth factor1 levels and to assess thyroid function 3 days before surgery. Hypopituitarism was defined as partial or complete loss of the hypothalamic-pituitary axis (10). Growth hormone deficiency was diagnosed on the basis of low serum insulin-like growth factor-1 levels or an insulin tolerance test (growth hormone peak $<10 \mu \mathrm{g} / \mathrm{l}$ ) (11). Gonadotropin deficiency was defined as low or inappropriate follicle stimulating hormone and luteinizing hormone levels with low testosterone levels (12). Central hypothyroidism was defined as low or inappropriate thyrotropin levels in the presence of low free T4 (FT4) levels (12). Adrenocorticotropic Hormone (ACTH) deficiency was defined by a peak cortisol value after provocative testing (insulin hypoglycemia test and glucagon or short synacthen test) of $<18 \mathrm{ng} /$ $\mathrm{ml}$ or a baseline plasma cortisol level of $<3 \mathrm{ng} / \mathrm{ml}$ (13).

BMI was expressed as the SD score based on the references established by Hui et al. (14). Obesity was defined as a BMI greater than 2 SDs above the gender- and age-specific mean; severe obesity was also expressed as a binary variable, defined by a BMI more than 3 SD above the age- and gender-specific mean. The influence of sex, preoperative obesity, tumor type, the presence of hypopituitarism or hydrocephalus, and PHS was analyzed as potential predictive factors.

\section{Follow-Up}

Follow-up data were obtained from patients' regular follow-up office visits and/or by contacting the patients and their families. Follow-up monitoring was performed at regular intervals based on clinical status, imaging findings, and the physician's preference. The median follow-up period was $32.16 \pm 22.25$ months (range 3-68 months). This study was approved by the Committee on Human Research at the Southern Medical University.

\section{Statistical Analysis}

The reporting of the present study closely follows the guidelines of the Transparent Reporting of a multivariable prediction model for Individual Prognosis Or Diagnosis (TRIPOD) statement (15). We developed the risk model based on the development cohort. First, we selected meaningful clinical risk factors by logistic regression with backward elimination. Candidate predictor variables that were statistically significant $(P<0.1)$ in univariate logistic regression models were considered for inclusion in the backward elimination multiple regression. In this step, the incremental change in the $c$ statistic was used to quantify the contribution to risk reclassification of each variable. We then devised a risk score (16) based on the final predictive model. The predictive accuracy of the risk score was assessed by both discrimination, as measured by the $c$ statistic (17), and calibration, as evaluated by the Hosmer-Lemeshow $\chi^{2}$-statistic (18) and a calibration plot (plot of observed proportions vs. predicted 
Table 1. Characteristics of patients included in the development and validation cohorts

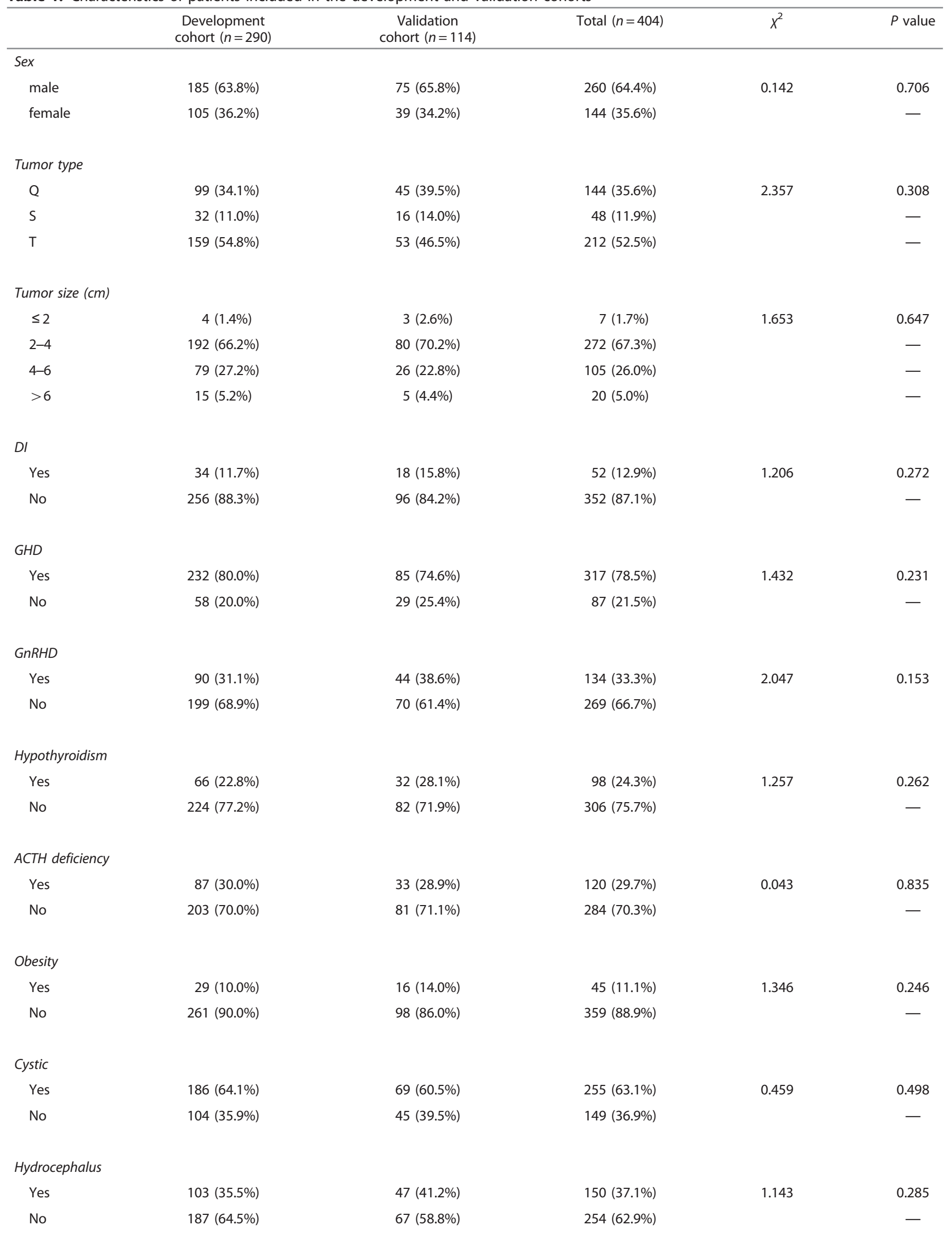




\section{Articles | ${ }_{\text {Li et al. }}$}

Table 1 Continued

\begin{tabular}{|c|c|c|c|c|c|}
\hline & $\begin{array}{c}\text { Development } \\
\text { cohort }(n=290)\end{array}$ & $\begin{array}{c}\text { Validation } \\
\text { cohort }(n=114)\end{array}$ & Total $(n=404)$ & $x^{2}$ & $P$ value \\
\hline \multicolumn{6}{|c|}{ Calcification } \\
\hline No & 42 (14.5\%) & $23(20.2 \%)$ & 65 (16.1\%) & & - \\
\hline \multicolumn{6}{|l|}{ PHS } \\
\hline 0 & 117 (40.3\%) & $54(47.4 \%)$ & $171(42.3 \%)$ & 2.667 & 0.264 \\
\hline 1 & 143 (49.3\%) & 53 (46.5\%) & $196(48.5 \%)$ & & - \\
\hline 2 & 30 (10.3\%) & 7 (6.1\%) & 37 (9.2\%) & & - \\
\hline
\end{tabular}

DI, diabetes insipidus; GHD, growth hormone deficiency; GnRHD, gonadotropin deficiency; PHS, preoperative hypothalamic status.

probabilities). The bootstrapping technique was used to adjust for overfitting and overoptimistic model performance. Finally, external validation of the risk score was performed to assess the robustness of the model.

\section{RESULTS}

The flowchart of participant enrollment is presented in Figure 1. Of the 404 participants, 65 (16.1\%) had developed severe obesity at the last visit. A total of $47(16.2 \%)$ participants in the development cohort and 18 (15.8\%) participants in the validation cohort developed severe obesity after surgery. Table 1 shows the baseline characteristics of eligible participants in the development and validation cohorts, as recorded at enrollment. The baseline characteristics of the validation cohort were generally similar to those of the development cohort, although the validation cohort was drawn from an independent group of participants. Neither significant nonlinearity nor significant interactions were found (Table 1).

\section{Risk Score Development}

Predictive models that included preoperative obesity, hydrocephalus, and PHS (Tables 2 and 3) as variables yielded c statistics of $0.524,0.612$, and 0.745 , respectively. Scores based on the final risk prediction model are presented in Table 4. The estimated predicted probability of developing severe obesity during follow-up observation after surgery in a patient with craniopharyngioma according to the proposed risk score is expressed as:

$$
P=\frac{1}{1+e^{-(-3.7269+0.3885 \times \text { score })}},
$$

where -3.7269 and 0.3885 are the intercept and slope coefficients of the regression, respectively. The total risk score ranges from a minimum value of 0 (lowest risk) to a maximum value of 16 (highest risk), with corresponding estimated predicted probabilities of developing severe obesity ranging from 2.4 to $92.3 \%$ (Table 4). The optimism-corrected c statistic of the risk score in the development cohort, computed using the bootstrapping technique, was 0.820 .
To illustrate the application of the risk score, consider the example of a 10-year-old girl with type $\mathrm{T}$ craniopharyngioma, hydrocephalus, obesity, and a PHS score of 1 . On the basis of the scoring system illustrated in Table 4 , her total risk score is $3.5+4.5+0.5=8$, and the estimated predicted probability that she will develop severe obesity is $35.0 \%$, as indicated by Table 5. An easy-to-use online tool was developed, whereby an individual's underlying risk of severe obesity can be estimated by entering the individual's characteristics.

\section{Risk Score Validation}

The $\mathrm{c}$ statistic of the risk score in the independent validation cohort was 0.821 . The receiver operating characteristic curves for the development and validation cohorts and by tumor type are shown in Figure 3. The calibration plots for both cohorts show close agreement between the predicted and observed risk of severe obesity, with no apparent over- or under-prediction (Figure 4). Calibration plots were constructed for both cohorts; the Hosmer-Lemeshow $\chi^{2}$ values in the development and validation cohorts were $\chi^{2}=0.384 \quad(P=0.944) \quad$ and $\quad \chi^{2}=1.361 \quad(P=0.715)$, respectively. The frequency of severe obesity was similar in the development and validation cohorts.

\section{Clinical Implications of the Risk Score}

We developed a clinical risk score based on tumor origin and obesity-related factors, as presented in Table 2. To enhance the clinical utility of the risk score, we used the quartiles of estimated risk to classify patients into three categories: low risk ( $0-5$ points), moderate risk (5-10 points), and high risk (10-16 points; Figure 5). The frequency of obesity in the development cohort and the validation cohort was 9.3 and 9.5\%, respectively, in the low-risk group, 31.1 and $40.0 \%$ in the moderate-risk group, and 63.9 and $66.7 \%$ in the high-risk group. As Figure 5 shows, the risk of developing severe obesity was highly and positively associated with the risk scores $(P$ for trend $<0.001)$.

The generated risk score was also correlated with the presence and stage of severe obesity. The score was significantly higher in those patients who developed severe 
Table 2. Baseline characteristics of patients in the development cohort who developed severe obesity vs. those who did not

\begin{tabular}{|c|c|c|c|c|c|}
\hline & $\begin{array}{c}\text { No severe } \\
\text { obesity }(n=243)\end{array}$ & $\begin{array}{l}\text { Severe obesity } \\
\quad(n=47)\end{array}$ & Total $n=290$ & $x^{2}$ & $P$ value \\
\hline \multicolumn{6}{|l|}{ Sex } \\
\hline Female & 86 (35.4\%) & 19 (40.4\%) & $105(36.2 \%)$ & & - \\
\hline S & 27 (11.1\%) & 5 (10.6\%) & 32 (11.0\%) & & - \\
\hline $\mathrm{T}$ & $129(53.1 \%)$ & $30(63.8 \%)$ & $159(54.8 \%)$ & & - \\
\hline \multicolumn{6}{|c|}{ Tumor size $(\mathrm{cm})$} \\
\hline$>6$ & 11 (4.5\%) & 4 (8.5\%) & 15 (5.2\%) & & - \\
\hline \multicolumn{6}{|l|}{ DI } \\
\hline Yes & $30(12.3 \%)$ & $4(8.5 \%)$ & 34 (11.7\%) & 0.560 & 0.454 \\
\hline No & $213(87.7 \%)$ & 43 (91.5\%) & $256(88.3 \%)$ & & - \\
\hline \multicolumn{6}{|l|}{$G H D$} \\
\hline Yes & 194 (79.8\%) & 38 (80.9\%) & $232(80.0 \%)$ & 0.025 & 0.873 \\
\hline No & 49 (20.2\%) & 9 (19.1\%) & $58(20.0 \%)$ & & - \\
\hline Yes & $53(21.8 \%)$ & $13(27.7 \%)$ & 66 (22.8\%) & 0.766 & 0.381 \\
\hline No & $190(78.2 \%)$ & 34 (72.3\%) & $224(77.2 \%)$ & & - \\
\hline \multicolumn{6}{|c|}{ ACTH deficiency } \\
\hline Yes & 69 (28.4\%) & $18(38.3 \%)$ & 87 (30.0\%) & 1.839 & 0.175 \\
\hline No & $174(71.6 \%)$ & $29(61.7 \%)$ & $203(70.0 \%)$ & & - \\
\hline \multicolumn{6}{|l|}{ Obesity } \\
\hline Yes & 12 (4.9\%) & 17 (36.2\%) & 29 (10.0\%) & 42.684 & 0.000 \\
\hline No & 231 (95.1\%) & 30 (63.8\%) & $261(90.0 \%)$ & & - \\
\hline \multicolumn{6}{|l|}{ Cystic } \\
\hline Yes & 187 (77.0\%) & 39 (83.0\%) & $226(77.9 \%)$ & 0.831 & 0.362 \\
\hline No & $56(23.0 \%)$ & $8(17.0 \%)$ & $64(22.1 \%)$ & & - \\
\hline \multicolumn{6}{|c|}{ Hydrocephalus } \\
\hline Yes & 73 (30.0\%) & $30(63.8 \%)$ & $103(35.5 \%)$ & 19.632 & 0.000 \\
\hline
\end{tabular}




\section{Articles | Li et al.}

Table 2 Continued

\begin{tabular}{|c|c|c|c|c|c|}
\hline & $\begin{array}{c}\text { No severe } \\
\text { obesity }(n=243)\end{array}$ & $\begin{array}{l}\text { Severe obesity } \\
\qquad(n=47)\end{array}$ & Total $n=290$ & $x^{2}$ & $P$ value \\
\hline No & $170(70.0 \%)$ & 17 (36.2\%) & 187 (64.5\%) & & - \\
\hline \multicolumn{6}{|c|}{ Calcification } \\
\hline Yes & 209 (86.0\%) & $39(83.0 \%)$ & 248 (85.5\%) & 0.292 & 0.589 \\
\hline No & 34 (14.0\%) & 8 (17.0\%) & $42(14.5 \%)$ & & - \\
\hline \multicolumn{6}{|l|}{ PHS } \\
\hline 0 & 105 (43.2\%) & $12(25.5 \%)$ & 117 (40.3\%) & 34.398 & 0.000 \\
\hline 1 & $124(51.0 \%)$ & 19 (40.4\%) & $143(49.3 \%)$ & & - \\
\hline 2 & $14(5.8 \%)$ & $16(34.0 \%)$ & 30 (10.3\%) & & - \\
\hline
\end{tabular}

Table 3. Univariate and multivariate logistic regression analyses of candidate risk factors for severe obesity (development cohort, $n=290$ )

\begin{tabular}{|c|c|c|c|c|c|c|}
\hline & \multicolumn{3}{|c|}{ Univariate analysis } & \multicolumn{3}{|c|}{ Multiplicity analysis } \\
\hline & OR & $95 \% \mathrm{Cl}$ & $P$ value & OR & $95 \% \mathrm{Cl}$ & $P$ value \\
\hline Obesity yes-no & 0.09 & $0.04 \sim 0.21$ & 0.000 & 5.76 & $1.37 \sim 24.30$ & 0.017 \\
\hline PHS 1-2 & 0.13 & $0.06 \sim 0.32$ & 0.000 & 2.21 & $1.22 \sim 20.35$ & 0.032 \\
\hline GnRHD yes-no & 1.26 & $0.54 \sim 2.93$ & 0.590 & & & \\
\hline Hypothyroidism yes-no & 0.73 & $0.36 \sim 1.48$ & 0.382 & & & \\
\hline DI yes-no & 2.17 & $0.90 \sim 5.24$ & 0.086 & & & \\
\hline Calcification yes-no & 1.26 & $0.54 \sim 2.93$ & 0.590 & & & \\
\hline Size $2-4 \mathrm{~cm}-4-6 \mathrm{~cm}$ & 1.15 & $0.56 \sim 2.36$ & 0.696 & & & \\
\hline Size $2-4 \mathrm{~cm}-<2 \mathrm{~cm}$ & 5.86 & $0.79 \sim 43.31$ & 0.083 & & & \\
\hline Size $2-4 \mathrm{~cm}->6 \mathrm{~cm}$ & 2.13 & $0.63 \sim 7.16$ & 0.222 & & & \\
\hline Type Type Q-Type T & 0.60 & $0.29 \sim 1.22$ & 0.16 & & & \\
\hline Type Type S-Type T & 0.80 & $0.28 \sim 2.24$ & 0.67 & & & \\
\hline
\end{tabular}

$\mathrm{Cl}$, confidence interval; DI, diabetes insipidus; GHD, growth hormone deficiency; GnRHD, gonadotropin deficiency; OR, odds ratio; PHS, preoperative hypothalamic status.

obesity than in those who did not, and a higher risk score was observed in patients with more severe obesity $(P<0.001)$. Furthermore, we developed a clinical score based on the clinical model with obesity-related factors, as presented in Table 2. Internal validation of the risk score was performed on the development data set using the bootstrap method, followed by external validation with the validation data set; the $c$ statistics for the internal and external validation were 0.820 (95\% confidence interval (CI) $0.751-0.889$ ) and 0.821 (95\% CI 0.699-0.942), respectively. The tabulation of the risk score for each variable is shown in Table 4. The receiver operating characteristic curves and calibration plots, as well as risk categories, are presented in Figures 3 and 5.

\section{DISCUSSION}

Hypothalamic obesity is a frequent complication of craniopharyngiomas and is refractory to current management options $(1,4,6,8)$. We developed and validated a clinical scoring system to identify PC patients at a very high or very low risk of developing severe obesity after surgery, before a 
Table 4. Risk scores for single risk factors associated with severe obesity in pediatric craniopharyngioma patients

\begin{tabular}{llc}
\hline Risk factor & Score & C statistic for single variable \\
\hline $\begin{array}{l}\text { Hydrocephalus } \\
\text { No }\end{array}$ & 0 & $0.612(0.550-0.675)$ \\
Yes & 3.5 & \\
Obesity & & \\
No & 0 & $0.524(0.482-0.566)$ \\
Yes & 4.5 & \\
PHS & & \\
0 & 0 & $0.745(0.662-0.829)$ \\
1 & 0.5 & \\
2 & 8 & \\
\hline PHS, preoperative hypothalamic status. &
\end{tabular}

Table 5. Predicted risk of severe obesity in pediatric craniopharyngioma patients based on the risk score model

\begin{tabular}{lc}
\hline Total risk score & Predicted risk (\%) \\
\hline 0 & 2.4 \\
0.5 & 2.8 \\
3.5 & 8.6 \\
4 & 10.2 \\
4.5 & 12.1 \\
5 & 14.4 \\
8 & 35.0 \\
8.5 & 39.5 \\
11.5 & 67.7 \\
12.5 & 75.6 \\
16 & 92.3 \\
\hline
\end{tabular}

detectable change in serum creatinine. The risk score was derived from a risk model including three clinical factors. To our knowledge, this is the first clinical scoring system derived and validated for early prediction of severe obesity in PC patients that incorporates clinical risk factors.

\section{Factors Influencing Severe Obesity}

Obesity has a major impact on quality of life in patients with craniopharyngioma (4-6). We observed a wide variation in the BMI of these patients, both in our data and in the literature, suggesting that preoperative obesity, tumor type, hydrocephalus, PHS, and severe obesity are closely correlated.

Several predictive factors have been proposed to determine the risk of obesity (5-8), including tumor size, calcification, and solidity. In the present study of clinical outcomes in 404 cases of PC with total tumor removal, no significant difference was found in the frequency of severe obesity or prognosis by tumor size or tumor components. In contrast, different tumor growth patterns were demonstrated to be highly associated

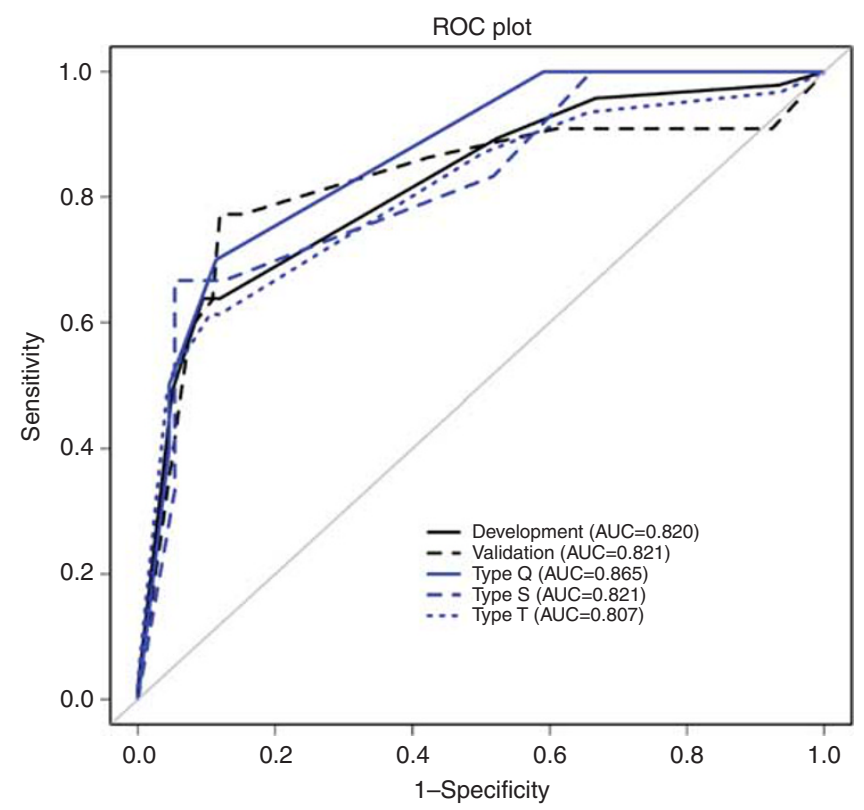

Figure 3. ROC curves showing the area under the curve for the prediction of severe obesity in PC patients. ROC curves showing the area under the curve for the risk score in the development cohort $(c=0.820)$, the validation cohort $(c=0.821)$, patients with type $\mathrm{Q}$ tumors in the development cohort $(c=0.865)$, patients with type $S$ tumors in the development cohort $(c=0.821)$, and patients with type T tumors in the development cohort $(c=0.807)$. PC, pediatric craniopharyngioma; $\mathrm{ROC}$, receiver operator characteristic.

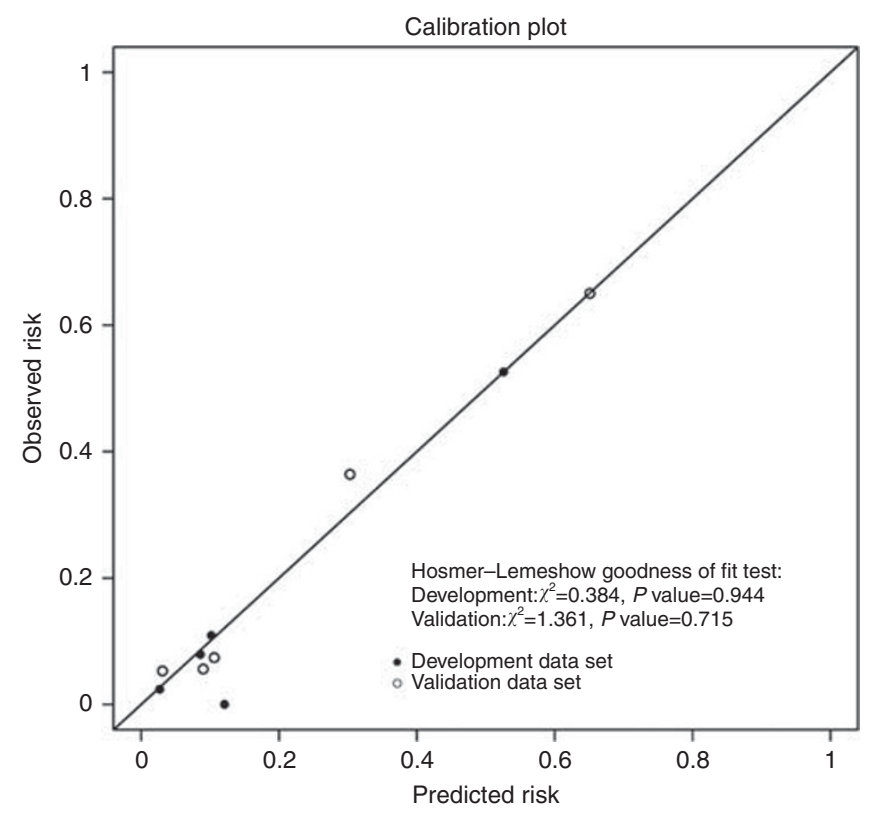

Figure 4. Calibration plot of observed vs. predicted risk of developing severe obesity during follow-up. The Hosmer-Lemeshow $X^{2}$-statistic is shown for the risk score in both the development and validation cohorts. Points and circles indicate the observed frequencies by decile of predicted probability.

with not only severe obesity but also clinical outcomes (Figure 3). A higher frequency of obesity, as well as decreased overall survival, progression-free survival, and poorer 


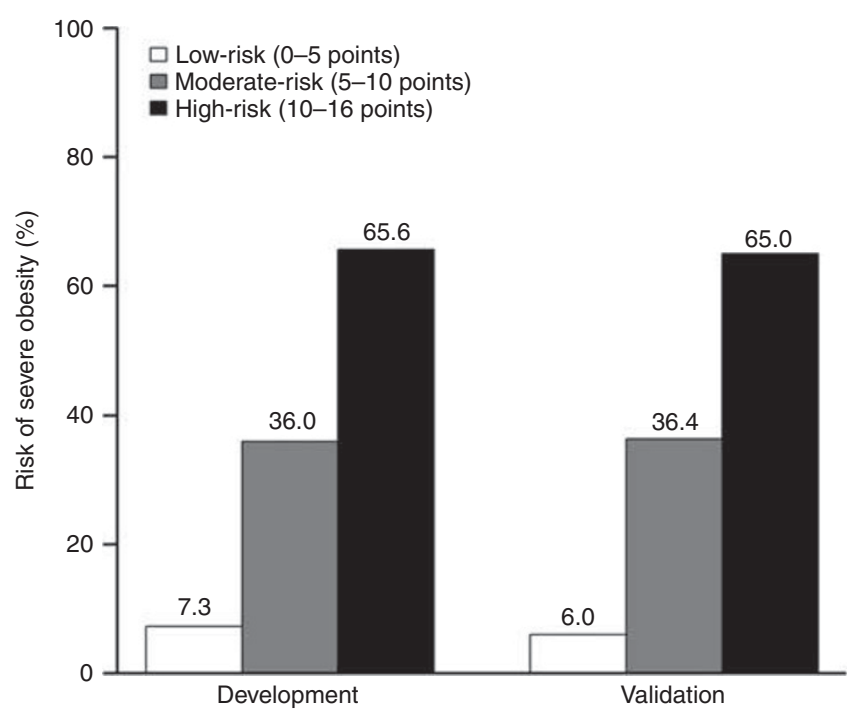

Figure 5. Risk levels according to the risk score in the development and validation cohorts. Scores were categorized into low-risk (0-5 points), moderate risk (5-10 points), and high risk (10-16 points) categories. A higher score was associated with a higher risk of developing severe obesity in PC patients; $P$ for trend $<0.001$.

outcomes, were evident in patients with type $\mathrm{T}$ tumors compared with the other two types of tumors. This finding was consistent with the literature.

Similar to other reports in the literature (19-24), the frequency of long-term severe obesity was $\sim 16.1 \%$ in our study group. The available data imply that, at least in some of these patients, type $\mathrm{T}$ tumors more frequently result in hyperinsulinemic obesity. In contrast, patients with type Q tumors presented with classical characteristics of hypopituitarism and apparent total growth hormone deficiency. This finding may be attributable to the differences in the location of the tumors. Unlike previous studies that defined tumor location as pre- and retrochiasmatic, or intrasellar and extrasellar (or both), we developed and used a morphological classification that considers both the tumor's pre-surgical appearance on imaging and its topographical relationship to membranous structures. Therefore, the accelerated weight increase is mainly due to the craniopharyngioma itself; in other words, tumor location may be the major factor influencing weight change.

In our series, the mean BMI SD score increased from 0.0 to 2.7 at 6 months to 2.4 at 1 year after surgery. Müller previously showed that preoperative BMI was the only independent predictor of long-term obesity in craniopharyngioma patients (8). Our data confirm these findings; children who developed hypothalamic obesity before surgery had a significant and rapid BMI increase over the first year after treatment, followed by stabilization, with no subsequent loss of weight; preoperative obesity seems to be a significant predictive factor for severe long-term obesity. In addition, patients who remained at a normal weight during follow-up showed a lower BMI SD score at the time of PC diagnosis $(8,25)$. Therefore, an increased BMI at diagnosis is another significant risk factor predictive of the development of severe obesity.

Hydrocephalus has been well documented as a predictor of poor outcomes following the surgical treatment of PCs. In the present study, obstructive hydrocephalus was rarely observed in patients with type $\mathrm{Q}$ and type $\mathrm{S}$ tumors, in spite of the large tumor size in some patients, whereas it was commonly observed in patients with type $\mathrm{T}$ tumors. These data suggest that the predictive value of hydrocephalus in PC surgery outcomes reflects differences in tumor location and growth patterns. The severity of obesity at presentation was significantly correlated with the presence of hydrocephalus, suggesting that the relative effects of hydrocephalus-causing PC tumors are intimately related to severe obesity.

The height of the tumor in the midline has been considered by some authors as a predictor of postoperative hypothalamic status (26). As Puget et al. (4) noted, a preoperative assessment indicating a loss of hypothalamic integrity might be refuted by postoperative magnetic resonance imaging showing reappearance of the floor of the third ventricle. The lack of reliability in assessing tumor invasion of hypothalamic structures on preoperative magnetic resonance imaging has also been documented by several other scholars. PHS is highly associated with the incidence rate of severe obesity postoperation, and the difference was statistically significant in our study. In our development cohort, for patients whose PHS $=0 \quad(n=117)$, we can find a boundary between tumor and brain tissue and remove tumor along the edge of its cyst membrane. A flat pituitary stalk can be seen behind the huge tumor cyst wall and reserved without being much disturbed. We believed that the hypothalamus tissue can be protected well in these patients and have the lowest incidence rate of postoperation severe obesity. For patients whose $\mathrm{PHS}=1$ $(n=147)$, tumors adhere ventricle tightly and gliosis is commonly found. Confrontational separation is needed, and aimless pulling that may cause damage to the hypothalamus and lead to severe obesity should be avoided. For patients whose PHS $=2(n=30)$, the boundary of tumor, gliosis, and hypothalamus tissue is unrecognized; a figure-like structure may be observed in the histologic section, and they have a high incidence for postoperative severe obesity. This finding could be partially explained by differences in tumor growth patterns; for example, infundibulotuberal tumors may disrupt the floor of the third ventricle at the offset, whereas infradiaphragmatic tumors usually arise adjacent to the floor of the third ventricle, even in very large-size tumors. The more serious the hypothalamic invasion by craniopharyngioma, the higher the rate of long-term obesity.

Accurate assessment of severe obesity is essential for the selection of appropriate therapy. In routine clinical practice, however, clinical outcomes vary among patients with the same tumor stages, suggesting that the present staging system is not adequate for the management of severe obesity. The present study derived and validated a potential clinical prediction tool rather than a decision rule, with the aim of helping the attending physician who will make the clinical 


\section{\begin{tabular}{l|l} 
A prospective cohort study in China & Articles
\end{tabular}}

decision. The factors incorporated in the proposed risk score are readily available data recorded on admission or from routine medical examinations.

\section{Highlights and Limitations of this Study}

Our study has several highlights. First, we derived, for the first time, a risk score for the early detection of PC patients at high risk of severe obesity. Second, the risk score showed high discriminative power, as well as high robustness and reproducibility, as shown by concordance between internal and external validation results. Third, in order to develop a prediction tool for the early detection of patients at risk of severe obesity after craniopharyngioma surgery, we excluded those who had recurrent tumors at admission, and we did not use these data in developing the risk score.

Our study also has certain limitations. First, its generalizability is limited, as all data were obtained from patients in China, and the distribution of clinical characteristics might be different in other areas. However, given the significant contribution of China to the worldwide burden of craniopharyngioma, it makes sense to perform an initial investigation in a Chinese population. Second, our findings remain to be confirmed by other independent studies. The question of whether the risk score predicts severe obesity due to craniopharyngiomas, or from other causes, remains to be addressed. Third, although the study participants were recruited from six hospitals located in five cities in China, the generalizability of the risk score to other population requires confirmation in further analyses.

\section{CONCLUSIONS}

Craniopharyngiomas are complex tumors associated with significant morbidity and mortality and require thoughtful treatment plans using a multidisciplinary and individualized approach. We developed and validated a novel risk score by incorporating major clinical risk factors to predict severe obesity in craniopharyngioma patients early. Individual risk prediction may assist clinicians in assessing the risk of severe obesity, which, in turn, would help them plan and initiate the most appropriate disease management for patients in a timely manner. In conclusion, this risk score can effectively predict obesity in PC patients and may facilitate patient counseling, decision-making regarding individualized adjuvant treatment, and follow-up monitoring.

\section{ACKNOWLEDGMENTS}

We thank Fan Jun, Xu Hai-yan, Mo Yi-Ping, and Duan Chongyang for the collection of data and care of patients enrolled in this study.

\section{STATEMENT OF FINANCIAL SUPPORT}

This research received grant from four fund projects in the public, including the National Nature Science Foundation of China (No. 81402759) and the Natural Science Foundation of Guangdong Province (No. 2015A030310279), Science and Technology Development, Southern Medical University (No. KJ20161126), and President Foundation of Nanfang Hospital, Southern Medical University (No. 2014B020).
Disclosure: We declare that we have no financial and personal relationships with other people or organizations that can inappropriately influence our work; there is no professional or other personal interest of any nature or kind in any product, service, and/or company that could be construed as influencing the position presented in, or the review of, the manuscript entitled "Risk Score for the Prediction of Severe Obesity in Pediatric Craniopharyngiomas: Relative to Tumor Origin".

\section{REFERENCES}

1. Van Effenterre R, Boch AL. Craniopharyngioma in adults and children: a study of 122 surgical cases. J Neurosurg 2002;97:3-11.

2. Yasargil MG, Curcic M, Kis M, et al. Total removal of craniopharyngiomas. Approaches and long-term results in 144 patients. J Neurosurg 1990;73:3-11.

3. Hoffman HJ. Surgical management of craniopharyngioma. Pediatr Neurosurg 1994;21 (Suppl 1): 44-9.

4. Puget S, Garnett M, Wray A, et al. Pediatric craniopharyngiomas: classification and treatment according to the degree of hypothalamic involvement. J Neurosurg 2007;106 (1 Suppl): 3-12.

5. Sterkenburg AS, Hoffmann A, Reichel J, et al. Nuchal skinfold thickness: a novel parameter for assessment of body composition in childhood craniopharyngioma. J Clin Endocrinol Metab 2016;101:4922-30.

6. Mortini P, Gagliardi F, Bailo M, et al. Magnetic resonance imaging as predictor of functional outcome in craniopharyngiomas. Endocrine 2016;51:148-62.

7. Olsson DS, Andersson E, Bryngelsson IL, et al. Excess mortality and morbidity in patients with craniopharyngioma, especially in patients with childhood onset: a population-based study in Sweden. J Clin Endocrinol Metab 2015;100:467-74.

8. Müller HL, Bueb K, Bartels U, et al. Obesity after childhood craniopharyngioma-German multicenter study on pre-operative risk factors and quality of life. Klin Padiatr 2001;213:244-9.

9. Qi S, Lu Y, Pan J, et al. Anatomic relations of the arachnoidea around the pituitary stalk: relevance for surgical removal of craniopharyngiomas. Acta Neurochir 2010;153:785-96.

10. Koulouri O, Auldin MA, Agarwal R, et al. Diagnosis and treatment of hypothyroidism in TSH deficiency compared to primary thyroid disease: pituitary patients are at risk of under-replacement with levothyroxine. Clin Endocrinol 2011;74:744-9.

11. Schmidt IL, Lahner H, Mann K, et al. Evaluation of the corticotropinreleasing hormone test and Basal serum cortisol in comparison to the insulin tolerance test in patients with hypothalamic-pituitary-adrenal disease. J Clin Endocrinol Metab 2003;88:4193-8.

12. Kazlauskaite R, Evans AT, Villabona CV, et al. Corticotropin tests for hypothalamic-pituitary-adrenal insufficiency: a meta analysis. J Clin Endocrinol Metab 2008;93:4245-53.

13. Colao A, Lombardi G. Growth-hormone and prolactin excess. Lancet 1998;352:1455-61.

14. Li H, Ji CY, Zong XN, et al. Hight and weight standardized growth dIans for Chhlese children and adolescents aged 0 to 18 years. Chin J Pediatr 2009;47:487-92.

15. Collins GS, Reitsma JB, Altman DG, et al. Transparent reporting of a multivariable prediction model for individual prognosis or diagnosis (TRIPOD):the TRIPOD statement. Br Med J 2015;350:g7594.

16. Sullivan LM, Massaro JM, D'Agostino RB. Presentation of multivariate data for clinical use: the Framingham Study risk score functions. Stat Med 2004;23:1631-60.

17. Cook NR. Statistical evaluation of prognostic versus diagnostic models: beyond the ROC curve. Clin Chem 2008;54:17-23.

18. Hosmer DW Jr, Lemeshow S, Sturdivant RX.. Applied Logistic Regression. New York: John Wiley \& Sons Inc, 2013.

19. Gautier A, Godbout A, Grosheny C, et al. Craniopharyngioma Study Group: Markers of recurrence and long-term morbidity in craniopharygioma: a systematic analysis of 171 patients. J Clin Endocrinol Metab 2012;97:1258-67.

20. Bülow B, Attewell R, Hagmar L, et al. Postoperative prognosis in craniopharyngioma with respect to cardiovascular mortality, survival, and tumour recurrence. J Clin Endocrinol Metab 1998;11:3897-904.

21. Manley PE, McKendrick K, McGillicudy M, et al. Sleep dysfunction in long term survivors of craniopharyngioma. J Neurooncol 2012;108:543-9. 


\section{Articles | Li et al.}

22. Müller HL, Handwerker G, Wollny B, et al. Melatonin secretion and increased daytime sleepiness in childhood craniopharyngioma patients. J Clin Endocrinol Metab 2002;87:3993-6.

23. Watson $\mathrm{SE}, \mathrm{Li} \mathrm{Z}, \mathrm{Tu} \mathrm{W}$, et al. Obstructive sleep apnoea in obese adolescents and cardiometabolic risk markers. Pediatr Obes 2014;9:471-7.

24. Verhulst SL, Schrauwen N, Haentjens D, et al. Sleep disordered breathing in overweight and obese children and adolescents: prevalence, characteristics and the role of fat distribution. Arch Dis Child 2007;92: 205-8.

25. Qi S, Peng J, Pan J, et al. Growth and weight of children with craniopharyngiomas based on the tumour location and growth pattern. J Clin Neurosci 2013;20:1702-8.

26. Ammirati M, Samii M, Sephernia A. Surgery of large retrochiasmatic craniopharyngiomas in children. Childs Nerv Syst 1990;6:13-7. 